The Surrogate Method - An Indirect Approach to Compound-Nucleus Reactions

J. Escher, L. Ahle, L. Bernstein, J. Burke, J. A. Church, F. Dietrich, C. Forssen, R. Hoffman, V. Gueorguiev

April 20, 2005

21st Winter Workshop on Nuclear Dynamics

Breckenridge, $\mathrm{CO}$, United States

February 5, 2005 through February 12, 2005 
This document was prepared as an account of work sponsored by an agency of the United States Government. Neither the United States Government nor the University of California nor any of their employees, makes any warranty, express or implied, or assumes any legal liability or responsibility for the accuracy, completeness, or usefulness of any information, apparatus, product, or process disclosed, or represents that its use would not infringe privately owned rights. Reference herein to any specific commercial product, process, or service by trade name, trademark, manufacturer, or otherwise, does not necessarily constitute or imply its endorsement, recommendation, or favoring by the United States Government or the University of California. The views and opinions of authors expressed herein do not necessarily state or reflect those of the United States Government or the University of California, and shall not be used for advertising or product endorsement purposes. 
Proc. 21st Winter Workshop on

Nuclear Dynamics (2005) 000-000 21st Winter Workshop on Nuclear Dynamics

Breckenridge, Colorado, USA

February 5-12, 2005

\title{
The Surrogate Method - An Indirect Approach to compound-nucleus reactions
}

\author{
J. Escher*, L. Ahle, L. Bernstein, J. Burke, J.A. Church, F. Diet- \\ rich, C. Forssén, R. Hoffman, and V. Gueorguiev \\ Lawrence Livermore National Laboratory, P.O. Box 808, L-414, Livermore, \\ CA 94551, USA
}

\begin{abstract}
An indirect method for determining cross sections for reactions proceeding through a compound nucleus is presented. Exploring indirect approaches for obtaining reaction cross sections is important since a large number of reactions relevant to astrophysics cannot be measured with currently available techniques. Of particular importance are reactions involving unstable nuclei. Some applications of the Surrogate nuclear reactions method are considered and challenges that need to be addressed are outlined.
\end{abstract}

Keywords: statistical compound-nucleus reactions, indirect methods, unstable nuclei, s process

PACS: 24.10.-i, 24.60.Dr, 25.40.Lw, 25.85.Ec

\section{Introduction}

Over the past 75 years we have acquired a basic, but incomplete, understanding of the processes that generate the energy in stars such as our sun, that drive the evolution of stars and that are responsible for the synthesis of the elements [1]. New astrophysical observations and recent progress in experimental techniques, coupled with an emerging generation of sophisticated models of astrophysical phenomena, present a unique opportunity for significant advances in our knowledge of the cosmos $[2,3]$. Directly linked to our understanding of astronomical objects and phenomena is an understanding of nuclear reactions, as these reactions synthesize elements, generate energy, and influence stellar evolution. Unfortunately, a large number of nuclear reactions relevant to astrophysics cannot be easily determined in the laboratory. Direct measurements encounter a variety of difficulties: The low-energy regime relevant for astrophysical reactions is often inaccessible; cross sections for charged-particle reactions become vanishingly small as the relative energy of the colliding nuclei decreases; and electron screening effects, which differ in labora- 
tory and stellar environments, are not completely understood. Furthermore, many reactions involve unstable nuclear species which are too difficult to produce with currently available experimental techniques or too short-lived to serve as targets in present-day set-ups.

In order to overcome the experimental limitations, various indirect methods have been proposed in recent years. Coulomb Dissociation [4], e.g., has been used to extract cross sections for radiative-capture reactions, $A(a, \gamma) B$. In this approach, the Coulomb field of a highly charged target provides a virtual photon, which is absorbed by the projectile $B$. The cross section of the breakup $X(B, A a) X$ is much larger than the capture cross section and can be related to the latter via the principle of detailed balance. The ANC (Asymptotic Normalization Coefficient) Method [5] has been explored for low-energy radiative-capture reactions $A(a, \gamma) B$ which are dominated by processes occurring far outside the nuclear radius. The cross section of such a reaction depends on the asymptotic behaviour of the overlap function $I_{A a}^{B}$ for $B \rightarrow A+a$. The radial shape of $I_{A a}^{B}$ is well known, and its normalization, the ANC, can be determined via a peripheral transfer reaction that involves the same asymptotic overlap, e.g. $d+A \rightarrow b+B$, where $d=a+b$ and $B=A+a$. The Trojan-Horse method [6] provides a mechanism for circumventing the Coulomb barrier which is responsible for the very small cross sections in low-energy twobody reactions with charged projectiles, $A(a, b) B$. It does so by selecting a reaction $d+A \rightarrow b+B+c$ with $d=a+c$ and kinematic conditions such that $c$ can be considered a spectator in the reaction between $a$ and $A$ ('quasi-free scattering'). An approximate expression for the cross section of the three-body reaction then provides a link to the two-body reaction of interest and allows one to extract the energy-dependence of the latter. Thus it becomes possible to extrapolate absolute measurements carried out at higher energies to the relevant low-energy regime.

The focus of this contribution is an indirect method that complements the above approaches, the Surrogate Nuclear Reactions method. The Surrogate method is potentially very valuable for studying two-step reactions on unstable targets. It combines experiment with reaction theory to obtain cross sections for reactions that proceed through a compound nucleus. A simple version of the Surrogate idea was already used in the 1970s to estimate neutron-induced fission cross sections from transfer reactions. More recently, this approach was refined and applications to other reactions are now being considered, such as $(n, \gamma)$ reactions on s-process branch points. The Surrogate concept is outlined in the next section. The astrophysical motivation for the current efforts is given in Section 3. Theoretical and experimental challenges for the Surrogate approach are summarized in Section 4. Some concluding remarks follow in Section 5.

\section{The Surrogate Idea}

The Surrogate nuclear reaction technique is an indirect method for determining the cross section for a particular type of "desired" reaction, namely a two-step reaction, 
$a+A \rightarrow B^{*} \rightarrow c+C$, that proceeds through a compound nuclear state $B^{*}$, a highly excited state in statistical equilibrium (see Figure 1). The formalism appropriate for describing compound-nucleus reactions is the statistical Hauser-Feshbach theory (see, e.g., chapter 10 of Ref. [7]).

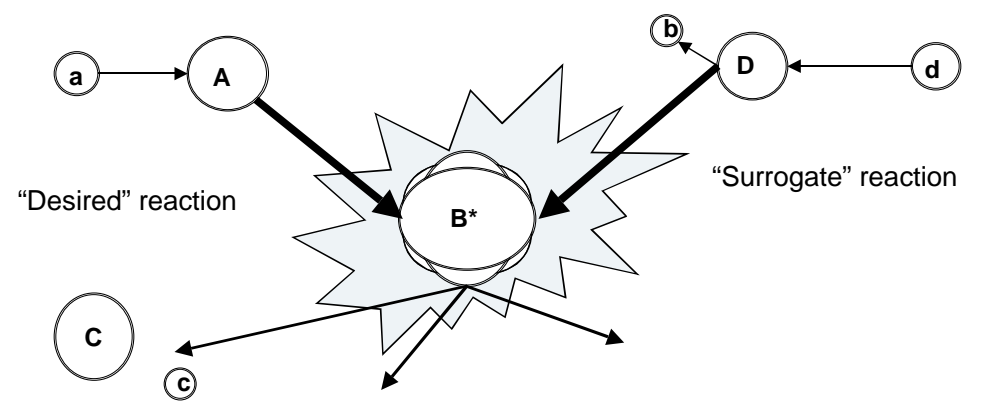

Fig. 1. Schematic representation of the Surrogate reaction mechanism. The basic idea of the Surrogate approach is to replace the first step of the desired reaction by an alternative ("Surrogate") reaction that populates the same compound nucleus. The subsequent decay of the compound nucleus into the relevant channel can then be measured and used to extract the desired cross section.

Formation and decay of a compound nucleus $(\mathrm{CN})$ are, by definition, independent of each other (for each angular momentum and parity value). In such situations, the cross section for the "desired" reaction can be (somewhat schematically) expressed as

$$
\sigma_{\alpha \chi}(E)=\sum_{J, \pi} \sigma_{\alpha}^{C N}(E, J, \pi) G_{\chi}^{C N}(E, J, \pi) .
$$

Here $\alpha$ denotes the entrance channel $a+A$ and $\chi$ represents the relevant exit channel $c+C$; $E$ is the excitation energy of the compound nucleus. In many cases the formation cross section $\sigma_{\alpha}^{C N}=\sigma\left(a+A \rightarrow B^{*}\right)$ can be calculated to a reasonable accuracy by using optical potentials, while the theoretical decay probabilities $G_{\chi}^{C N}$ for the different channels $\chi$ are often quite uncertain. The objective of the Surrogate method is to determine or constrain these decay probabilities experimentally.

In a Surrogate experiment, the compound nucleus $B^{*}$ is produced via an alternative ("Surrogate"), direct reaction $d+D \rightarrow b+B^{*}$ and the decay of $B^{*}$ is observed in coincidence with the outgoing particle $b$. The probability for forming $B^{*}$ in the Surrogate reaction (with specific values for the excitation energy $E$, angular momentum $J$, and parity $\pi$ ) is $F_{\delta}^{C N}(E, J, \pi)$, where $\delta$ refers to the entrance channel $d+D$. The quantity

$$
P_{\delta \chi}(E)=\sum_{J, \pi} F_{\delta}^{C N}(E, J, \pi) G_{\chi}^{C N}(E, J, \pi),
$$

which gives the probability that the compound nucleus $B^{*}$ was formed with energy $E$ and decayed into channel $\chi$, can be obtained experimentally. The direct-reaction 
probabilities $F_{\delta}^{C N}(E, J, \pi)$ have to be determined theoretically, so that the decay probabilites $G_{\chi}^{C N}(E, J, \pi)$ can be extracted from the measurements and, subsequently, inserted in equation (1) to yield the desired reaction cross section.

While the Surrogate method is very general and can in principle be employed to determine cross sections for all types of (two-step) reactions on a large variety of nuclei, its greatest potential value lies in applications to reactions on unstable isotopes.

\subsection{Early Surrogate Experiments}

The Surrogate approach was used in the 1970s, albeit in a very simplistic manner, to extract $(n, f)$ cross sections for various actinides from transfer reactions with $t$ and ${ }^{3} \mathrm{He}$ projectiles on neighboring nuclei, followed by fission [8]. Measured fission probabilities, $P_{f}$, were simply multiplied by an estimated cross section for the formation of the compound nucleus in the neutron-induced reaction of interest: $\sigma_{(n, f)} \approx \sigma_{n}^{C N} P_{f}$. While the resulting $(n, f)$ cross section estimates agreed with direct measurements (where available) to about 10-20\% for incident neutron energies above about $1 \mathrm{MeV}$, it resulted in serious discrepancies below $1 \mathrm{MeV}$, which were attributed to i) large uncertainties in the low-energy optical-model calculations employed, and ii) the neglect of the difference in the angular-momentum populations of the compound nucleus in the Surrogate (direct) and "desired" (neutron-induced) reactions (see Section 4).

In addition to fission measurements, some early experiments were carried out to assess the feasibility of using the Surrogate technique to determine cross sections for $(n, \alpha)$ and $(n, p)$ reactions on nuclei in the mass-90 region [9]. These experiments highlighted further issues that needed to be addressed in order to extract reliable cross sections from Surrogate measurements. In particular, the effects of projectile break-up in the Surrogate reaction needed to be estimated and ambiguities in identifying the reaction sequence in some reactions needed to be resolved. It was concluded that a comprehensive theory effort would be required to eliminate these uncertainties.

\subsection{The Surrogate Method - Revisited}

Recently, Younes and Britt [10] revisited the Surrogate $(t, p f),\left({ }^{3} \mathrm{He}, d f\right)$, and $\left({ }^{3} \mathrm{He}, t f\right)$ measurements from the 1970s. They employed a simple direct-reaction model to account for the angular-momentum population difference between neutron-induced and direct reactions and used improved optical-model calculations to obtain $(n, f)$ cross sections for various $\mathrm{Th}, \mathrm{U}, \mathrm{Np}, \mathrm{Pu}$, and Am isotopes. For the benchmark case ${ }^{235} \mathrm{U}$, they obtained significant improvements over the early Surrogate work. In addition to reproducing the fission cross section for the $J^{\pi}=7 / 2^{-}$ground state of ${ }^{235} \mathrm{U}$, Younes and Britt were able to estimate the fission cross section for the isomeric $1 / 2^{+}$state at $77 \mathrm{eV}$, which to date has not been measured directly. In general, the $(\mathrm{n}, \mathrm{f})$ cross sections deduced by Younes and Britt for various actinide 
nuclei agree with direct measurements to within $\pm 10 \%$ for $E_{n} \gtrsim 1 \mathrm{MeV}\left[E_{n} \gtrsim 2\right.$ $\mathrm{MeV}]$ when obtained from Surrogate (t,pf) $\left[\left({ }^{3} \mathrm{He}, x f\right)\right]$ data; at lower energies the discrepancies are about $20 \%$.

\section{Surrogate Reactions and Astrophysical Applications}

Cross sections for unstable nuclei are not only very difficult to measure, they also play an important role for addressing some of the most compelling questions of basic science. Among the unanswered mysteries about the nature and evolution of our universe is the origin of the heavy elements [3]. Much effort is currently being devoted to exploring nuclear processes, such as the s and r processes ("slow" and "rapid" neutron-capture processes), and astrophysical environments that can produce the elements between iron and uranium. Of particular interest in the context of the s process are branch points, unstable nuclei that are produced in the s process with a life time long enough to allow the s process to proceed by either neutron capture or $\beta$ decay [11]. A crucial ingredient for determining the probability of one path dominating over the other is the associated neutron-capture cross section. The resulting calculated abundance patterns can be compared to data from stellar spectroscopy or analyses from presolar dust grains. Such comparisons impose stringent tests on available s-process models and provide valuable information on the physical conditions under which the s process takes place. In addition, accurate and precise knowledge of the s process provides important constraints for the $r$ process. Since little is known about the astrophysical site(s) and the actual path of the r process, its abundances are inferred by subtracting calculated s-process abundances from measured total abundances [12].

In some sense, s-process branch points provide an excellent opportunity for testing the Surrogate approach. The "desired" reaction, to be obtained from a Surrogate experiment, is a neutron-capture reaction on an unstable nucleus ${ }^{A} Z$ which is located in or very close to the valley of stability. Both its ${ }^{A-1} Z$ neighbor and the intermediate nucleus ${ }^{A+1} Z$ that is formed in the neutron-capture process are usually stable. Consequently, there are various options for forming the relevant compound nucleus in a Surrogate (direct) reaction, e.g. a 2-neutron pickup reaction on ${ }^{A-1} Z$ or inelastic scattering on ${ }^{A+1} Z$ can be considered. On the other hand, the neutron energies relevant to the s process are very low. Current s-process scenarios have characteristic temperatures corresponding to neutron energies $E_{n}=8$ and 23 $\mathrm{keV}$ for the production of the elements between $\mathrm{Zr}$ and $\mathrm{Bi}$ in the main s-process component, and $E_{n}=26$ and $91 \mathrm{keV}$ for the production of the elements between Fe and $\mathrm{Zr}$ in the weak s-process component [11]. The low energies imply that very little angular momentum is transferred from the neutron to the target, while the angularmomentum transfer in the Surrogate reaction can be much larger, thus leading to significant differences between the compound-nucleus populations obtained in the two different reactions (see " $J^{\pi}$ population mismatch" in Section 4). Furthermore, recent progress in astronomical observations and stellar modeling translates into a 
need for high-precision data on neutron-capture rates. In some cases a $1 \%$ accuracy is desired [11]. While it is highly unlikely that this level of accuracy can be reached in an indirect approach such as the Surrogate method, one has to recall that there are are key reactions for which no experiments exist and whose calculated cross sections have very large uncertainties. In particular, reactions on unstable target nuclei have to be determined indirectly (or with radioactive beams in inverse kinematics). In the following, we will consider some of the issues that have to be resolved before the Surrogate approach can be employed to extract cross sections for $(n, \gamma)$ reactions of importance to the s process.

\section{Challenges for the Surrogate Approach}

In order to assess whether the Surrogate technique can be used to reliably determine low-energy $(n, \gamma)$ cross sections, various theoretical and experimental challenges have to be addressed. The most obvious issue that needs to be studied is the so-called " $J{ }^{\pi}$ population mismatch": A compound nucleus is, by definition, a quantum-mechanical system in statistical equilibrium, and as such has no memory of its formation process. Nevertheless, constants of motion, such as angular momentum and parity, are conserved throughout the reaction. In general, the Surrogate reaction populates the states in the intermediate nucleus differently than the desired $a+A$ channel, i.e. the weights $F_{\delta}^{C N}(E, J, \pi)$ by which the decay probabilites $G_{\chi}^{C N}(E, J, \pi)$ are multiplied in equation (2), are different from the formation probabilities $\sigma_{\alpha}^{C N}(E, J, \pi) / \sum_{J^{\prime}, \pi^{\prime}} \sigma_{\alpha}^{C N}\left(E, J^{\prime}, \pi^{\prime}\right)$ of equation (1), and depend on the direct reaction under consideration. In the early work it was assumed that the experimental conditions were such that the Weisskopf-Ewing limit applied, i.e. the decay probabilites were independent of $J^{\pi}$ and the cross section of the reaction could be written as a simple product $\sigma_{\alpha \chi}(E) \approx \sigma_{\alpha}^{C N}(E) G_{\chi}^{C N}(E)$. The more recent work by Younes and Britt demonstrates the importance of accounting for the " $J^{\pi}$ population mismatch".

In order to identify reactions that can possibly serve as Surrogates for a particular desired reaction, it is important to know the $J^{\pi}$ populations that are obtained in various direct reactions (stripping, pick-up, inelastic scattering), as well as the dependence of these populations on projectile, target, excitation energy, angle of outgoing particle, etc. This is a nontrivial task since a proper treatment of direct reactions leading to highly excited states in the intermediate nucleus involves a description of particle transfers, and inelastic scattering, to unbound states.

It is furthermore important to study how the $J^{\pi}$ populations that can be obtained in the various reactions affect the decay of a compound nucleus. More specifically, if there is a significant " $J$ " population mismatch" between the Surrogate and

"desired" reactions, one has to investigate whether the Surrogate measurement can provide meaningful constraints for the cross section of the "desired" reaction.

To obtain the experimental decay probabilities $P_{\delta \chi}(E)$ for a particular exit channel $\chi=C+c$, it is essential to identify the final reaction product(s) in that chan- 
nel. For some applications of the Surrogate technique, this identification involves detecting fission fragments. In other cases, the identification involves measuring characteristic $\gamma$ rays from the de-excitation of the desired reaction product $C$. In order to convert the measured $\gamma$-ray intensities to channel probabilities $P_{\delta \chi}(E)$, a model for the de-excitation process is needed, which, in turn, requires a proper description of structural properties of the remnant $C$, such as level densities, branching ratios between discrete levels, and internal conversion rates.

Another important issue to be investigated in the context of Surrogate reactions is the role of pre-equilibrium reactions: Central to the Surrogate method is the assumption that the formation and decay of the intermediate nuclear state - in both the "desired" and the Surrogate reaction - are independent of each other (apart from conserving constants of motion). This is only valid if the intermediate nucleus equilibrates (becomes a "compound" nucleus) before it decays into the final reaction products. Rapid ("pre-equilibrium") decay of the intermediate configuration before a compound nucleus can be formed would invalidate the Surrogate analysis. The probability that a compound nucleus is actually formed in a particular reaction needs to be estimated and the effects of pre-equilibrium decays on energy balances and $J^{\pi}$ populations have to be studied.

Finally, to establish the validity of the Surrogate approach, it is necessary to carry out experiments that provide appropriate benchmarks. Planning for such a benchmark experiment is underway: the reaction $\alpha+{ }^{102} \mathrm{Ru} \rightarrow \alpha^{\prime}+{ }^{102} \mathrm{Ru}^{*}$ will be used as a Surrogate for $n+{ }^{101} \mathrm{Ru} \rightarrow{ }^{102} \mathrm{Ru}^{*}$. Observing the decay ${ }^{102} \mathrm{Ru}^{*} \rightarrow{ }^{102} \mathrm{Ru}$, by $\gamma$-ray emission, in coincidence with the outgoing $\alpha$ particle allows one to infer the cross section for $n+{ }^{101} \mathrm{Ru} \rightarrow \gamma+{ }^{102} \mathrm{Ru}$, which can then be compared to available data from direct measurements.

\section{Concluding Remarks}

Indirect methods play an important role for obtaining reaction cross sections of interest to astrophysics. A method which aims at extracting cross sections for reactions proceeding through a compound nucleus has been presented. Possible applications of the method to reactions relevant to the astrophysical s process have been considered and the main challenges for the method have been outlined. Clearly, all indirect approaches have disadvantages. It is therefore important to carefully study and understand the strengths and limitations of each method. While the desired accuracy may not be in immediate reach, it might still be possible to determine a particular cross section in several independent indirect ways and thus to gain confidence in the results and to obtain important constraints for the physics under investigation. 
J. Escher et al.

\section{Acknowledgment(s)}

This work was performed under the auspices of the U.S. Department of Energy by the University of California, Lawrence Livermore National Laboratory (LLNL) under contract No. W-7405-Eng-48. The project (04-ERD-057) is funded by the Laboratory Directed Research and Development Program at LLNL.

\section{Note}

* E-mail: escher1@llnl.gov

\section{References}

1. C.E. Rolfs and W.S. Rodney, Cauldrons in the Cosmos, University of Chicago Press, Chicago, 1988.

2. DoE/NSF Nuclear Science Advisory Committee, Opportunities in Nuclear Science - A Long-range Plan for the Next Decade, April 2002; F.-K. Thielemann et al., Prog. Part. Nucl. Phys. 46 (2001) 5;

3. Board on Physics and Astronomy, National Research Council 2003, Connecting Quarks to the Cosmos: Eleven Science Questions for the New Century, National Academies Press, Washington, D.C., 2003.

4. G. Baur, K. Hencken, and D. Trautmann, Prog. Part. Nucl. Phys. 51 (2003) 487; G. Baur and H. Rebel, Annu. Rev. Nucl. Part. Sci. 46 (1996) 321;

5. N.K. Timofeyuk, R.C. Johnson, and A.M. Mukhamedzhanov Phys. Rev. Lett. 91 (2003) 232501; L. Trache et al., Phys. Rev. C 69 (2004) 032802(R); L. Trache et al., Phys. Rev. Lett. 87 (2001) 271102; A. Azhari et al., Phys. Rev. Lett. 82 (1999) 3960; A.M. Mukhamedzhanov and R.E. Tribble, Phys. Rev. C 59 (1999) 3418; H. M. Xu et al., Phys. Rev. Lett. 73 (1994) 2027.

6. S. Typel and G. Baur, Ann. Phys. 305 (2003) 228; G. Baur and S. Typel, preprint nucl-th/0401054.

7. P. Fröbrich and R. Lipperheide, Theory of Nuclear Reactions, Oxford University Press, Oxford, U.K., 1996.

8. J.D. Cramer and H.C. Britt, Nucl. Sci. and Eng. 41 (1970) 177; J.D. Cramer and H.C. Britt, Phys. Rev. C 2 (1970) 2350; B.B. Back et al. , Phys. Rev. C 9 (1974) 1924; B.B. Back et al. , Phys. Rev. C 10 (1974) 1948; H.C. Britt and J.B. Wilhelmy, Nucl. Sci. and Eng. 72 (1979) 222.

9. H. C. Britt and J. B. Wilhelmy, private communication.

10. W. Younes W and H.C. Britt, Phys. Rev. C 67 (2003) 024610; W. Younes W and H.C. Britt, Phys. Rev. C 68 (2003) 034610.

11. F. Käppeler and A. Mengoni, Nucl. Phys. A (in press); F. Käppeler, Prog. Part. Nucl. Phys. 43 (1999) 419; M. Busso, R. Gallino, and G.J. Wasserburg, Ann. Rev. Astron. Astrophys. 37 (1999).

12. Y.-Z. Qian, Prog. Part. Nucl. Phys. 50 (2003) 153; J.J. Cowan, F.-K. Thielemann, and J.W. Truran, Phys. Rep. 208 (1991) 267. 